\title{
An Unusual Presentation of Growing Skull Fracture with Sutural Diastasis
}

\author{
Gaurav Sharma ${ }^{1}$ Gaurav Jain ${ }^{1} \quad$ Jitendra Shekhawat ${ }^{1} \quad$ Sanjeev Chopra ${ }^{1}$ Virendra Deo Sinha ${ }^{1}$ \\ ${ }^{1}$ Department of Neurosurgery, SMS Medical College, Jaipur, \\ Address for correspondence Dr. Gaurav Jain, MBBS, DNB, \\ Rajasthan, India \\ Department of Neurosurgery, SMS Medical College, Jaipur 302004 \\ Rajasthan, India (e-mail: drgauravjain9@gmail.com).
}

Indian J Neurotrauma:2020;17:53-56
Abstract
Keywords
- craniocerebral erosions
- dural laceration
- growing skull fracture
- leptomeningeal cyst
- sutural diastasis

Growing skull fracture (GSF) is a rare complication of childhood skull fractures, which is caused by progressive diastatic enlargement of the fracture line. Progressive swelling is the most common presenting feature. The most common site is the parietal region. Sutural diastasis is a rare site for development of GSF. Early treatment is must as they cause delayed onset neurological deficit and cranial asymmetry. The aim of this report is to discuss an unusual presentation of GSF with sutural diastasis and review of literature.

\section{Introduction}

Growing skull fracture (GSF), leptomeningeal cysts and craniocerebral erosions, is a rare entity that occurs after severe head trauma during the first 3 years of life (particularly in infancy). The reported incidence is 0.05 to $1.0 \%$ among all childhood skull fractures and occurrence after 8 years of age is rare. ${ }^{1,2}$ They occur as a result of linear fractures which are often neglected by both parents and treating physicians. ${ }^{1,3,4}$ The child may present with progressive scalp swelling and widening of fracture margins associated with herniation of meninges as well as brain tissue. They warrant prompt treatment as they might cause delayed onset neurological deficit, seizure, and cranial asymmetry. The most common site is parietal bone. GSF in frontal bone, which is associated with sutural diastasis, is rare. ${ }^{5}$ Here, we present a rare case of GSF in the frontal region with sagittal suture diastasis and left frontal bone fracture. The fracture line was reaching up to the coronal suture on the left side, with a large underlying dural defect through which the brain tissue was herniating.

\section{Case Report}

A 1-year-old child was brought to our department with complaints of gradually progressing swelling over the frontal region ( - Fig. 1a). Attendants gave history of child falling from the bed 8 months ago, following which patient developed left frontal swelling. They consulted the local physician and were managed conservatively; however, no investigation was done. Swelling progressively increased over the last 8 months for which parents again consulted the local practitioners, but recorded no improvement. Patient had no history of vomiting, seizure, or neurological deficit.

On examination, there was soft, cystic, globular, compressible, and nontender swelling in the bifrontal region, extending anteriorly to supraorbital rim, laterally to superior temporal lines, and posteriorly up to coronal suture. Swelling was transilluminant with cough impulse present. The anterior fontanelle was open and lax. There was no neurological deficit.

Noncontrast computed tomography (CT) of head (three-dimensional [3D] reconstruction) was done, which was suggestive of left frontal bone fracture, reaching up to coronal suture on left side, with diastasis of coronal suture indicated by arrows in $\mathbf{- F i g}$. $\mathbf{1 b}$.

Bony defect was also seen in the anterior midline, as marked by arrow in - Fig. 1c. Intraoperatively, cerebrospinal fluid (CSF) was coming out of this defect with herniation of brain tissue.

Patient underwent surgery and a bicoronal skin incision was made. After elevating the skin flap, a large underlying cystic swelling of size $10 \times 8 \mathrm{~cm}$ was seen, which was aspirated ( - Fig. 2a). Aspirate was clearly suggestive of CSF. 


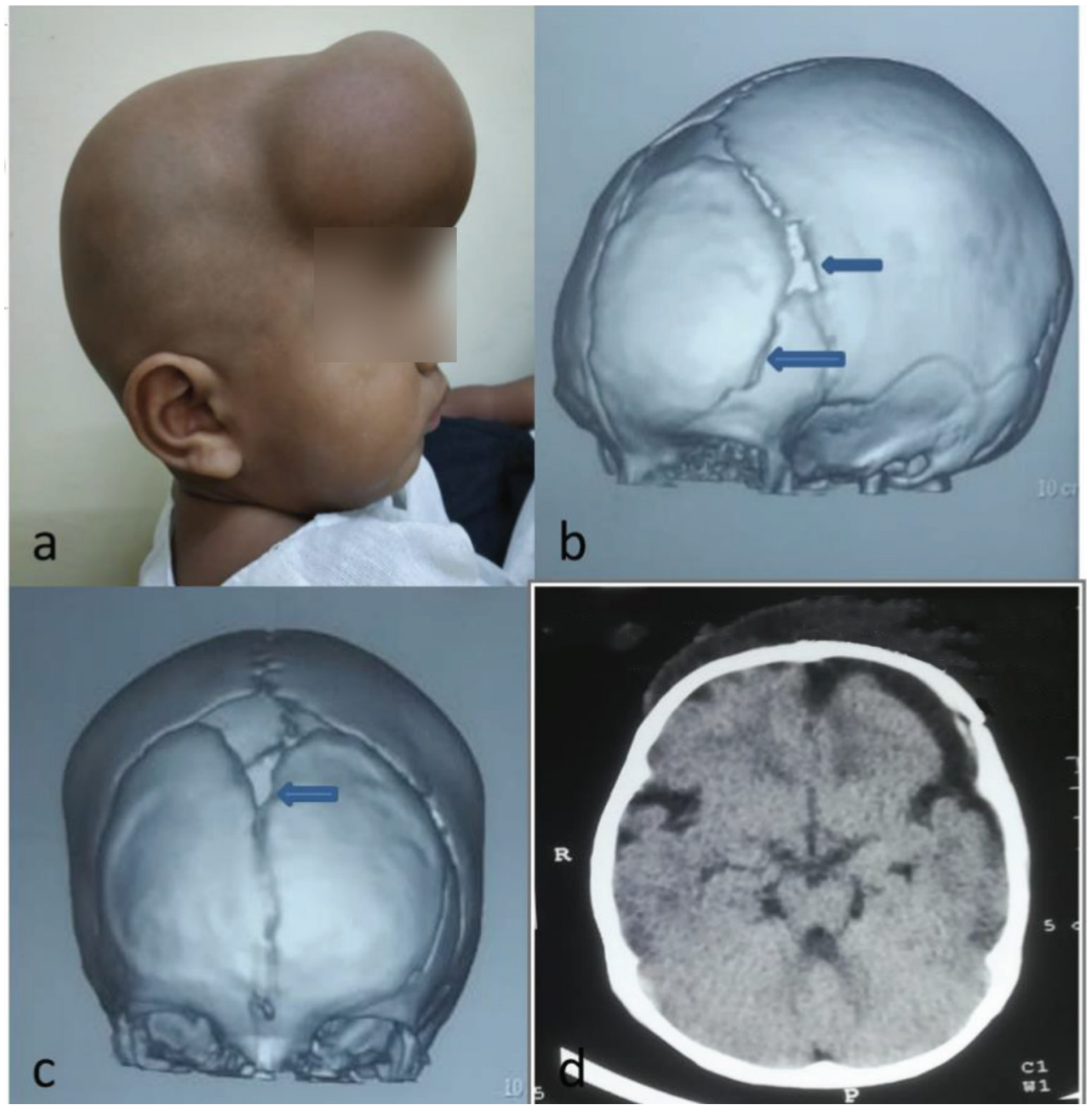

Fig. 1 (a) Child presented with globular swelling in frontal region. (b) Fracture line in left frontal bone, reaching up to left coronal suture. (c) Bony defect was also seen in anterior midline. Intraoperatively, CSF was coming out of this defect with herniation of brain tissue. (d) Axial cuts suggestive of fracture left frontal bone with subdural hygroma and hypodense extracalvarial collection over frontal bone. Abbreviation: CSF, cerebrospinal fluid.

The cyst wall was then opened, and the left frontal bone fracture with coronal as well as midline sutural diastasis were noted along with a midline bony defect of size $1 \times 1 \mathrm{~cm}$. Brain tissue was adherent and CSF was seen egressing through

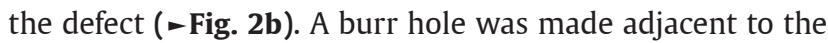
left frontal bone fracture site, and craniectomy was done to define normal dural margins. The dural defect was extending across the midline to right side, so craniectomy had to be extended toward the right side. Midline diastatic segment was elevated and the patient developed bleeding from superior sagittal sinus, due to tear in the midline dura while performing craniectomy, which was managed with oxidized cellulose and gelfoam application. Blood transfusion was done intraoperatively as the child developed hypotension. Dura was gently separated from the underlying brain and was then repaired using pericranial patch and fibrin glue in a watertight manner.

The bony defect could not be repaired in view of hemodynamic instability. Skin closure was done and the patient was shifted to the ICU postoperatively.
On day 6 postoperation, the patient developed swelling with signs of inflammation at the operated site. With suspicion of wound infection, the patient was taken up for wound irrigation. A drain was placed which was removed the next day. The patient improved gradually and was then discharged on day 10 postoperation. The patient came for follow-up in OPD on a regular basis, and after 2 months of follow-up, the patient had no swelling or CSF leak.

\section{Discussion}

GSF was first reported by Howship in 1816 . Since then, many studies have reported variable incidence rates of around 0.05 to $1.0 \%$ among all childhood skull fractures. In early childhood, brain volume increases rapidly, which is, in part, responsible for their development at the fracture site, along with a prerequisite of underlying dural laceration. Brain pulsations and raised intracranial pressure do not allow fractures to heal. There occurs invagination of arachnoid in fracture line, leading to leptomeningeal cyst formation and herniation 


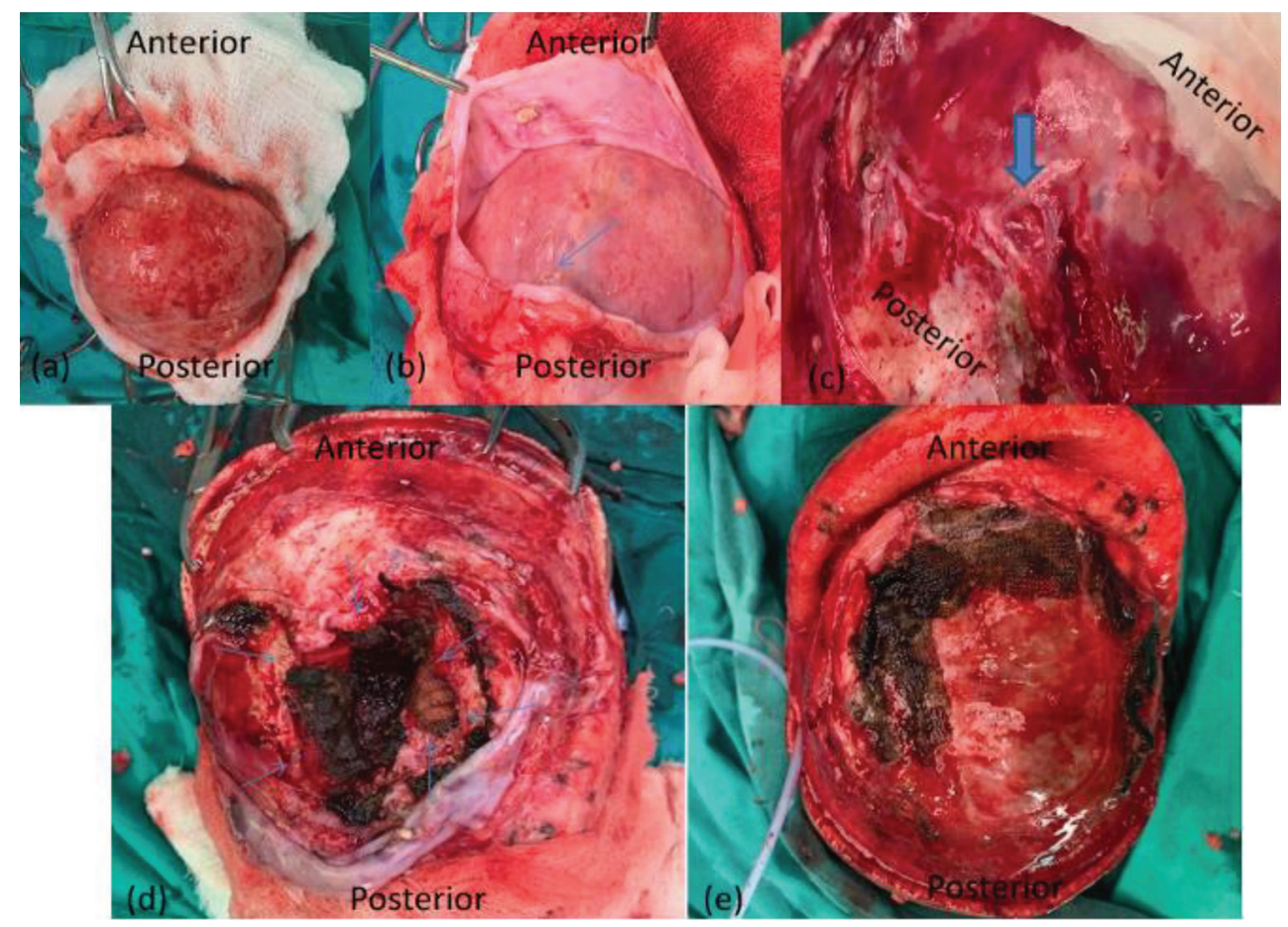

Fig. 2 (a) Large cystic collection seen in midline, extending bilaterally in frontal region after elevation of skin flap. (b) Midline defect seen after opening the cyst wall with CSF and brain coming out of midline sutural diastasis site. (c) Closer view of the bony defect seen before craniectomy. (d) A much larger dural defect was present, underlying the small bony defect (dural margins shown with arrows). (e) Watertight dural closure was done using pericranial patch and fibrin glue. Abbreviation: CSF, cerebrospinal fluid.

of brain tissue. Resorption of adjacent bone by way of continuous pressure from brain, and meningeal herniation through the gap, leads to further widening of fractured margins.

Fracture line with underlying brain damage is another risk factor for development of GSF, as it indicates significant trauma to cause dural laceration. It is almost always seen in GSFs, but is not a necessity for their development. ${ }^{6}$ There occurs cerebromeningeal cicatrix formation at the growing fracture site.

Leptomeningeal cysts are fluid-filled spaces between pia and arachnoid matter. They are not true cysts as they are not completely isolated from subarachnoid space.

Fracture diastasis of $>4 \mathrm{~mm}$ may be considered as a risk factor for development of GSF. $7{ }^{78}$ They occur mostly in the parietal region but association with cranial suture diastasis is rare.

All infants sustaining trauma to the head should undergo plain X-ray of skull, and if fracture is seen or suspected, CT scan of head is warranted.

On basis of CT appearance, GSF are classified into the following:

Type I-GSF with leptomeningeal cyst, which may be seen herniating through the skull defect into the subgaleal space. Type II-associated with underlying brain damage or gliosis. Type III-associated with porencephalic cyst.
Noncontrast CT scan of head with bony window is essential for diagnosis of GSF and its management.

Review of literature suggests children less than 3-years-old with cephalohematoma, underlying brain damage or bone diastasis $>4 \mathrm{~mm}$ on $\mathrm{CT}$, and seizures immediate to the injury are more susceptible to develop GSF. All such patients should undergo CT and, more importantly, magneticresonanceimaging(MRI)scansatinitialpresentation. CT scan is not sensitive enough to detect dural tear at initial presentation, whereas MRI has greater sensitivity. ${ }^{9-11}$ In developing countries, where MRI is not available, B ultrasound can be used for early dural tear detection. ${ }^{12}$

Patients are at risk of seizure, CSF leaks, and calvarial deformity. Prompt treatment is the key to prevent these complications, and delayed treatment makes the surgery more difficult. A well-planned and widely tailored craniectomy should be done to define the dural defect, which may be extended, if needed, to define dural margins well. Dural defect is generally found to be larger than the bony defect. ${ }^{13,14}$ Primary closure of dural defect should not be attempted, as this may lead to raised intracranial tension. Watertight closure using autologous graft and artificial dural patches is done, which should be confirmed by the Valsalva maneuver. Artificial dural patches should be avoided in the presence of infection. Skull defects should be closed by split thickness bone graft taken from appropriate site or rib graft. Epilepsy, hemiparesis, or other neurological deficits may not be 
always due to GSF; rather, they can be manifestations of initial brain injury. Therefore, they may not improve after GSF surgery.

Follow-up after head injury is very important to diagnose GSF. The parents should be informed about the possibility of GSF in such head injury cases. Warning signs such as persistent swelling or onset of new neurological deficit needs utmost attention.

\section{Conclusion}

Frontal region is an uncommon site for development of GSF, and association with sutural diastasis is even rare, as in our case. Any persistent or late-appearing swelling in the frontal region with history of head trauma should raise the suspicion of GSF.

Children sustaining head trauma due to any cause should be evaluated for skull fracture. Initially, skull X-ray should be done, and if indicated, head CT should be done. Even linear fracture of the skull bone should be managed with utmost attention. Early treatment of GSF is the key point because if delayed or left untreated, they might lead to significant morbidity.

\section{Authors' Contribution}

Contributor 1: Dr. Gaurav Sharma.

Contributor 2: Dr. Gaurav Jain.

Contributor 3: Dr. Jitendra Shekhawat.

Contributor 4: Dr. Sanjeev Chopra.

Contributor 5: Dr. Virendra Deo Sinha.

Department of Neurosurgery, Sawai Man Singh Medical

College, Jaipur, India.

All were involved in clinical assessment, management of the patient, and preparation of manuscript.

The manuscript has been read and approved by all the authors, and each author believes that the manuscript represents honest work.

\section{Conflict of Interest}

The authors report no conflicts of interest.

\section{References}

1 Vignes JR, Jeelani Nu, Dautheribes M, San-Galli F, Liguoro D. Cranioplasty for repair of a large bone defect in a growing skull fracture in children. J Craniomaxillofac Surg 2007; 35(3):185-188

2 Ziyal IM, Aydin Y, Türkmen CS, Salas E, Kaya AR, Ozveren F. The natural history of late diagnosed or untreated growing skull fractures: report on two cases. Acta Neurochir (Wien) 1998;140(7):651-654

3 Leung GK, Chan KH, Hung KN. Growing skull fracture in an adult nine years after blunt head trauma. J Clin Neurosci 2011; 18(6):855-857

4 Singla N, Gupta SK. The natural history of an untreated growing skull fracture: an unusual case. Pediatr Neurosurg 2010; 46(1):76-79

5 Khandelwal S, Sharma GL, Gopal S, Sakhi P. Growing skull fractures leptomeningeal cyst. Indian J Radiol Imaging 2002;12:485-486

6 Lende RA, Erickson TC. Growing skull fractures of childhood. J Neurosurg 1961;18:479-489

7 Gruber FH. Post-traumatic leptomeningeal cysts. Am J Roentgenol Radium Ther Nucl Med 1969;105(2):305-307

8 Taveras JM, Ransohoff J. Leptomeningeal cysts of the brain following trauma with erosion of the skull; a study of seven cases treated by surgery. J Neurosurg 1953;10(3):233-241

9 Husson B, Pariente D, Tammam S, Zerah M. The value of MRI in the early diagnosis of growing skull fracture. Pediatr Radiol 1996;26(10):744-747

10 Gallo P, Mazza C, Sala F. Intrauterine head stab wound injury resulting in a growing skull fracture: a case report and literature review. Childs Nerv Syst 2010;26(3):377-384

11 Hamamcioglu MK, Hicdonmez T, Kilincer C, Cobanoglu S. Large intradiploic growing skull fracture of the posterior fossa. Pediatr Radiol 2006;36(1):68-70

12 de P Djientcheu V, Njamnshi AK, Ongolo-Zogo P, et al. Growing skull fractures. Childs Nerv Syst 2006;22(7):721-725

13 Ersahin Y, Gülmen V, Palali I, Mutluer S. Growing skull fractures (craniocerebral erosion) Neurosurg Rev 2000;23(3):139-144

14 Singhal A, Steinbok P. Operative management of growing skull fractures: a technical note. Childs Nerv Syst 2008;24(5): 605-607 